\title{
Music Perception with Current Signal Processing Strategies for Cochlear Implants
}

\author{
Waldo Nogueira, Martin Haro, Perfecto Herrera, Xavier Serra \\ Music Technology Group, Pompeu Fabra University \\ Tanger Building, Roc de Boronat, 138 \\ +34935422000 \\ waldo.nogueira@upf.edu
}

\begin{abstract}
This work presents a brief review on hearing with cochlear implants with emphasis on music perception. Although speech perception in noise with cochlear implants is still the major challenge, music perception is becoming more and more important. Music can modulate emotions and stimulate the brain in different ways than speech, for this reason, music can impact in quality of life for cochlear implant users. In this paper we present traditional and new trends to improve the perception of pitch with cochlear implants as well as some signal processing methods that have been designed with the aim to improve music perception. Finally, a review of music evaluation methods will be presented.
\end{abstract}

\section{Keywords}

Cochlear Implant, Music, Signal Processing, Music Perception.

\section{INTRODUCTION}

Cochlear implants are implanted medical devices that can restore hearing to people with profound hearing loss [1]. Approximately more than 200,000 patients today have received cochlear implants worldwide. Speech recognition for cochlear implant recipients in noisy conditions and music perception are severely limited with these devices. A factor that determines hearing performance is the signal processing method used in cochlear implants. Therefore, a great deal of research is focused on designing new sound processing methods.

Generally a Cochlear Implants (CI) (Figure 1) consists of two parts: the external part and the internal part. The external part contains a microphone, a sound processor and a transmitting coil. The internal part is surgically implanted inside the patient's head and consists of a receiving coil or antenna, a decoder and stimulator, and the electrode array. Actual electrode arrays contain 8 to 22 electrodes. In actual commercial systems each electrode can only be stimulated at a fixed rate between $250 \mathrm{~Hz}$ and 2320 Hz. The rate of stimulation is the same for all electrodes. Compared to an intact natural cochlea, which contains thousands of hair cells performing an accurate temporal analysis of sound, CI systems still only provide reduced auditory information.

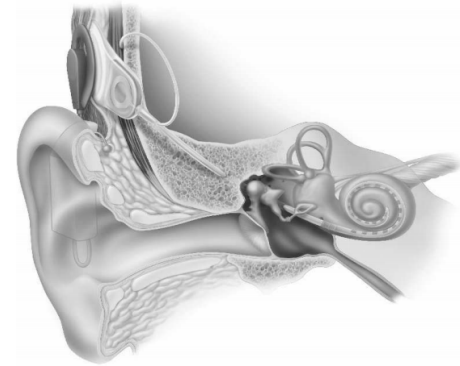

Figure 1: External and Internal parts of a Cochlear Implant System

In CIs the audio signal is picked up by the microphone and is processed in the digital sound processor. The sound processor decomposes the incoming sound into different frequency bands (sub-bands) and calculates the electric charge that has to be applied to each electrode depending on the energy of each frequency band. This information is then encoded and sent through the external transmitter-coil. The internal receiver takes the coded electrical signals and delivers them to the current source in the stimulator. Commonly, electrodes are activated with charge balanced symmetrical biphasic pulses to avoid charging and thus damage of tissues in the cochlea. When an electrode is activated, the auditory nerves close to that electrode will start to send action potentials to the brain. Therefore, the sound processor activates electrodes close to the base of the cochlea when the incoming sound contains high frequency components, and activates electrodes towards the apex of the cochlea when the sound contains low frequency components. Each electrode acts as a channel that conveys information of a determined frequency and bandwidth to the central mechanisms of the auditory system. This functionality mimics the action of the basilar membrane and the inner hair cells in the human auditory system.

Signal Processing and Audio coding strategies play a very important role in maximizing the user's overall communicative potential. Over the years different signal processing algorithms and CI strategies have been developed to mimic firing patterns inside the cochlea as naturally as possible [2]. Figure 2 shows a block diagram with the different processing stages used in a CI processor. The block diagram of Figure 1 is composed by two parts: the signal processing algorithms (which analyze and process the sound), and the CI sound coding strategy (which converts or codes the sound into electrical stimulation that is sent to the electrodes).

The analog signal is picked up by the microphone is digitized and is sent to a front-end processing block that tries to clean the noise 
from the signal using single and multichannel channel noise reduction techniques.

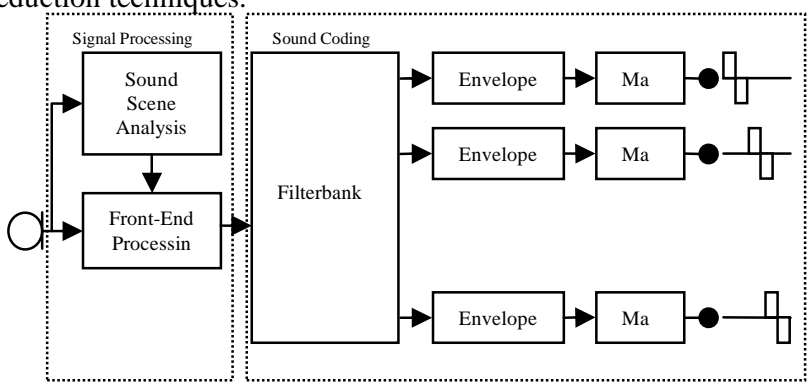

Figure 2: Block diagram showing the cochlear implant strategy composed by the signal processing and the sound coding stage. In this document the cochlear implant strategy refers to all algorithms applied in a cochlear implant system just after the signal is captured by the microphone. The signal processing part includes noise reduction, classification of the sound environment and adaptive gain control. The sound coding strategy is defined as the stage that converts the audio signal into electrical signals that are presented to the auditory nerve using electrodes (filterbank, envelope and map)

\section{Hearing with Cochlear Implants}

Users of cochlear implants do not hear as well as normal hearing listeners. Three possible reasons for this are, first, the degradation of sound perception derived from the channel interactions produced when the electrodes are stimulated [3]. Second, cochlear implant users obtain worse pitch perception in comparison to normal hearing listeners [4]. Third, cochlear implant systems use a simple model to mimic the functionality of a normal cochlea [5] [6].

Channel interaction can occur with simultaneous and nonsimultaneous stimulation. The activation of an electrode causes the stimulation of a large population of auditory nerves around this electrode, as fluids in the cochlea are relatively good conductors. This phenomenon is known as current spread. When more than one electrode stimulates the same or overlapping regions of the auditory nerve, the stimuli may be perceptually confused. This fact causes that underlying populations of auditory nerves would receive the combined stimulus and not the separate stimuli from each electrode. This limits the number of independent information channels conveyed to the central auditory mechanisms and can degrade speech recognition. Studies on normal hearing listeners have demonstrated that 10 independent bands are too few for speech recognition and 30 bands give no improvement in accuracy over 20 bands [4]. However, studies on cochlear implantees have shown that speech recognition scores do not improve as the number of electrodes are increased beyond four to seven [8]. One reason given to explain this, is that as the number of electrodes is increased, the probability for current field interactions between electrodes is also increased. This causes the information between electrodes not to be dependent. A negative correlation has been shown between electrical field interaction and speech recognition performance [3].

Pitch perception can be obtained by cochlear implant recipients using two known mechanisms [9]: The temporal pitch or pitch rate and the place pitch. The first mechanism is related to the temporal pattern of stimulation. The higher the frequency of stimulation, the higher is the pitch perceived. However, most patients do not perceive differences in the frequency of stimulation at individual electrodes as differences for rates of stimulation above $300 \mathrm{~Hz}$ [10]. Temporal pitch cues in cochlear implants have shown to provide a means for fundamental frequency discrimination [11] and melody recognition [9]. The fundamental frequency is important for speaker recognition and the improvement of speech intelligibility. For example in tonal languages (e.g. Cantonese, or Mandarin), changes of the pattern within a phonemic segment determine the lexical meaning. Cochlear implant users in countries with tone languages do not seem to derive the same benefits as individuals who speak nontonal languages [12].

The second mechanism, the place pitch, is related to the spatial pattern of stimulation. Electrodes located towards the base of the cochlea produce higher pitch sensations than electrodes located towards the apex of the cochlea. The resolution of place pitch might be limited by the low number of electrode contacts and the current spread produced in the cochlea when each electrode is activated. When the listening environment becomes challenging, increased spectral resolution is required to separate speech from noise or to distinguish multiple talkers [13]. It has been shown that increased spectral resolution is required to perceive harmonic pitch and to identify melodies and instruments [14]. As many as 100 bands of spectral resolution are required for music perception in normal hearing subjects [13]. Moreover, the ability for an accurate differentiation of place pitch information is also important for the perception of the fundamental frequency [11]. The simple modeling of the electrode-nerve interface in cochlear implants that is intended to substitute for the hair cells inside the cochlea is clearly not as sophisticated as a fully functional cochlea. Today's systems attempt to mimic thousands of nerve fibers using electrode arrays that contain, at most, 8 to 22 electrode contacts. Furthermore, the use of relatively low and fixed stimulation rates and the use of biphasic pulses for stimulation seems to produce non-natural responses of the auditory nerve in comparison to a normal cochlea [15]. Finally, large adaptive non-linear effects in loudness and phase relationships between different sections of the cochlea are still not modeled in actual cochlear implant devices [5] [6]. Therefore, it becomes apparent that the way in which these few electrodes are selected and activated by the signal processor plays a key role in helping cochlear implant subjects to improve sound perception.

\subsection{Music Perception with Cochlear Implants}

An improvement of music perception with hearing prostheses can lead to improved quality of life and improved sound perception abilities (including speech in noise). Many CI and hearing aid users are unsatisfied with their music perception after implantation. In order to increase the quality of life (QoL) for this population [16] [17] further research is needed on perception and enjoyment of music. Additionally, recent research indicates that musical experience modulates auditory functions in normal hearing listeners, leading to easier perception of speech in difficult listening situations or scenes. This finding implies that musical experience can positively affect the ability of understanding speech in noise. 
So far, it was believed that CIs cannot convey the fine temporal and frequency information to the auditory nerve to properly perceive music. The reason for this is the poor transmission of information from the electrodes to the auditory nerve [6]. Usually the electrodes inside the cochlea produce very large excitation patterns in the cochlea that difficult clear perception of frequencies with these devices. A better perception of frequencies, or pitch, might help to improve perception of music. Several studies investigating the different properties of music tried to identify the major difficulties for music perception with CI subjects [18] [19]. The outcome of these studies showed that rhythmical structures are perceived by CI users almost as good as by normal hearing listeners [20]. Melody recognition is a difficult task for adult CI recipients. The perception of pitch in CI subjects is worse than the sensitivity found in normal hearing subjects [21]. Pitch in CI recipients is important for melody recognition [22] and musical interval identification [18]. Normal listeners achieve pitch thresholds of around 0.2 semitones whereas CI users achieve between 2 to 7 semitones [23]. There is a relation between the bad pitch perception scores and the bad melody recognition with CI. Timbre perception, which is related to musical instrument identification, seems to perform similar to normal hearing. Therefore, CIs provide meaningful information for timbre cues, even though they might be different from what listeners were used to hear before implantation [18].

\subsection{Techniques to improve pitch perception}

Historically, the number of electrodes in a system has defined the number of spectral bands of stimulation [13]. Therefore, the spectral resolution was limited to the maximum number of electrodes. Given the fixed number of electrodes researchers have investigated mechanisms to enhance spectral resolution without adding additional electrodes. It has been reported [24] that additional pitch perceptions can be created by stimulating two electrodes at the same time. This technique is referred to as current steering (Figure 3). A study showed that the perception of pitch could be varied systematically by adjusting the proportion of current delivered simultaneously to two electrodes [25]. The additional pitches were perceived as one pitch rather than two separate pitch components and were intermediate to the pitches heard when either electrode was stimulated alone.

Further research in this field found that for most subjects, the loudness perception for either of the adjacent electrodes stimulated alone was equivalent to the loudness perception when the current delivered simultaneously to two electrodes was apportioned by linear interpolation [26]. Recently, a study on a larger group of CII and HiRes90k users [27] has estimated that the number of virtual channels or intermediate pitches along the electrode array range from 8 to 466 , with an average of 93 . It should be remarked that for cochlear implants with only one current source, the ability to steer current is not possible [13]. Researchers have found another mechanism that uses nonsimultaneous stimulation for producing pitch percepts that are intermediate to those evoked by the electrodes when stimulated alone [28]. Pitch percepts can be created when two neighboring electrodes are stimulated sequentially but at a high rate. In this case, the spread of the electrical field may cause stimulation on adjacent electrodes to overlap in time. However, it has been suggested that sequential or non-simultaneous dual electrode stimulation may be more complicated to control in terms of loudness as compared to simultaneous dual electrode stimulation. A strategy, termed SineEx, that uses the current steering technique to deliver frequency information has been designed [34]. In current steering, pairs of electrodes are stimulated simultaneously. SineEx models the audio signals with sinusoids and presents only the frequencies of these sinusoids using current steering. SineEx was designed parallel to the F120 strategy of Advanced Bionics. Both strategies are the first signal processing strategies implemented in a commercial device using the current steering technique. These strategies were evaluated in a chronic study comparing them to the standard and non-current steering strategy, HiRes. It seems that current steering strategies show a trend for improved frequency discrimination than non-current steering strategies.

To further evaluate sound performance with current steering strategies, a questionnaire was used to evaluate music and speech preference. The SineEx strategy obtained better speech naturalness and pleasantness. Music quality with SineEx was rated more clear, natural and pleasant than with Hires. Subjective tests have shown a preference for current steering strategies against sequential strategies.

The small improvements observed with current steering strategies might be caused by the fact that channel interactions in a cochlea might smear the information transmitted. Recently current focusing has been proposed to increase the number of distinct perceptual channels available to CI users by adjusting currents applied simultaneously or-non-simultaneously to multiple CI electrodes. New developments in CIs try to improve the electrode nerve interface by applying current steering and current focusing which allows a much finer transmission of frequencies from the electrodes to the nerve. Current focusing in CIs can be achieved by multi-polar stimulation ([29]), this technique has been proposed to confine better the electrical fields and improve speech and music perception with these devices. However, the potential of current steering and current focusing has to be further investigated. Recently the benefits of current focusing in pitch perception have been shown in patients ([30]).

The range of pitch sensations that can be evoked by electric stimulation of the cochlea in a cochlear implant was thought to be limited by the position of the most apical and basal electrodes in the cochlea. Recently a new technique has been developed to produce pitch sensations that are lower than the most apical electrode or higher than the most basal electrode. This technique has been termed Phantom. In 10 subjects it was shown that a shift of between 1 to 2 electrodes was possible [32].

Another method to extend pitch sensation beyond those obtained by the apical electrode in the cochlea has been presented in [31]. Their technique is based on the assumption that short phases are more effective (i.e. need less charge to evoke the same loudness) than longer phases and that anodic (positive) phases are more effective than cathodic (negative) ones [31]. They have shown that at low pulse rates, these stimuli elicit a lower place-pitch percept than symmetric pulses presented in monopolar or in bipolar mode and that they allow the subject to perceive increases in temporal pitch up to higher rates than for other intra-cochlear stimulation sites and/or pulse shapes.

More recently a new cochlear implant strategy based on Phantom technique and bandwidth extension towards the low frequencies has been developed and evaluated in CI users by a major manufacturer with the aim to improve music perception of these devices ([35]). A music questionnaire was designed to evaluate 
music perception with this strategy and compare its perception against a commercial current steering strategy called F120. Three music tokes were selected and presented through a loudspeaker and a subwoofer. Using both strategies, music was easy to follow, with a small advantage for Phantom. With Phantom music was perceived slightly more natural than with F120. The sound balance with Phantom was rated to be more neutral than with F120. Finally, the overall impression of music with Phantom was significantly better than with F120. These developments show the great interest of the industry in the field of music but also show the need to create better evaluation methods for music than just using subjective questionnaires.

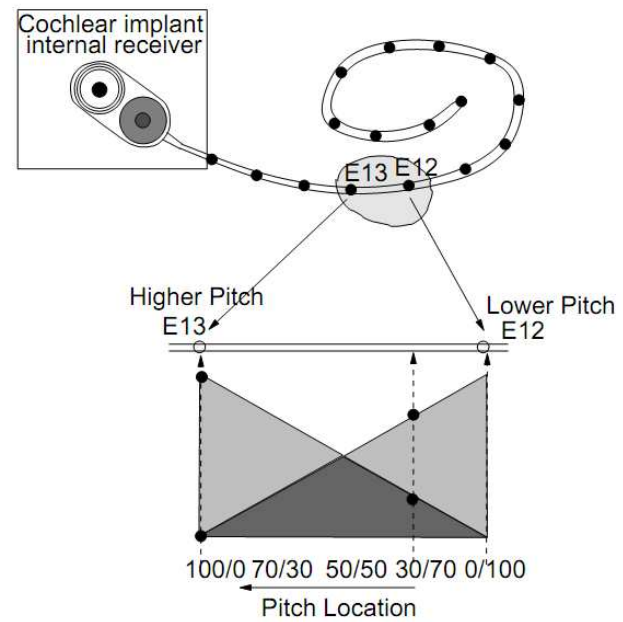

Figure 3: Current steering paradigm. Current is delivered to two electrodes simultaneously (El12 and El13). Changing the proportion of current between both electrodes it is possible to represent intermediate pitch perceptions. In the figure the proportions delivered to each electrode are represented by 100/0, 70/30, 50/50, 30/70, and 0/100

These new techniques to improve pitch perception are very promising and will allow the creation of new signal processing strategies that hopefully can enhance the perception of speech and music with cochlear implants.

\section{Evaluation methods to assess music perception in Cochlear Implants}

During the past decades many evaluation procedures have been developed in order to assess speech understanding with different signal processing algorithms in hearing prostheses. In terms of music perception however, there is a need to further investigate evaluation methods. This includes subjective questionnaires or psychophysical measures of basic properties of sound (including pitch, timbre, etc) and more cognitive processes (including music memory, emotion, etc). Few standardized test exists to quantify music perception in a clinically practical manner [23].

Typically subjective sound perception with new cochlear implant strategies is evaluated using questionnaires that assess how the user takes advantage of the CI in daily life. Usually, questionnaires ask questions regarding pleasantness, distinctness, naturalness, and overall perception of music.
Psychophysical measures of basic properties of sound have been also used to assess music perception outcomes with a CI. This includes tests to evaluate basic properties of sound like pitch and timbre and tests to evaluate features of music like instrument and number of instrument identification as well as melody recognition tasks.

However it has been found that in some cases music battery tests to predict performance with a CI do not necessarily correlate with the subjective enjoyment of music in daily life[33]. For this reason, there is a need to investigate new measures to evaluate music and their correlation to everyday perception of music using CIs. Just as an example, the perception of emotions through music and CIs might be an interesting topic to focus on.

Finally, music battery tests can be used to assess performance but probably can be also used by CI users directly to train themselves when hearing into music. Research has shown that although cochlear implant users generally find music to be less enjoyable following implantation, training may help some recipients to improve their music perception.

\section{Summary}

This paper has presented a brief overview on how a cochlear implant works and how hearing is perceived with these devices. Currently, a number of techniques and approaches are being proposed to improve pitch perception with cochlear implant users, including frequency extension, improved pitch coding through current steering and focusing as well as extension of temporal pitch. Improved pitch perception will hopefully lead to improved music perception with these devices. If successful, these approaches could improve the quality of life for implantees by improving communication and musical and environmental awareness. Moreover, the approach used to evaluate these new techniques will play a major role, the combination of subjective appreciation questionnaires and its correlation to more objective measures like pitch, timbre, melody or instrument identification tasks has to be better understood as well as the integration of emotion tests in order to assess how music is perceived in daily life.

\section{REFERENCES}

[1] B. S. Wilson, C. C. Finley, D. T. Lawson, R. D. Wolford, D. K. Eddington, and W. M. Rabinowitz, "Better speech recognition with cochlear implants," Nature, vol. 352, pp. 236-238, 1991

[2] P. C. Loizou, "Signal-processing techniques for CIs," IEEE Eng. Med. Biol. Mag., vol. 18, no. 3, pp. 34-46,1999.

[3] G. Stickney, "Analyses of speech processing strategies for cochlear implants and the effects of electrodes interaction," Phd dissertation, Universitiy of Texas at Dallas, 2001.

[4] J. Laneau, M. Moonen, and J. Wouters, "Factors affecting the use of noise-band vocoders as acoustic models for pitch perception in cochlear implants," Journal of the Acoustical Society of America, vol. 119, pp. 491-506, 2006.

[5] B. S.Wilson, R. S. Schatzer, E. A. Lopez-Poveda, X. Sun, D. T. Lawson, and R. D.Watford,"Two new directions in speech processor design for cochlear implants," Ear and Hearing, pp. 73-81, August 2005. 
[6] B. C. J. Moore, "Coding of sounds in the auditory system and its relevance to signal processing and coding in cochlear implants," Otology \& Neurotology, vol. 24, pp. 243-254, 2003.

[7] J. B. Allen, "How do humans process and recognize speech?," IEEE Transactions on speech and audio processing, vol. 2, no. 4, pp. 567-577, October 1994.

[8] K. Fishman, R. V. Shannon, and W. H. Slattery, "Speech recognition as a function of the number of electrodes used in the SPEAK cochlear implant speech processor," IEEE Transactions on speech and audio processing, vol. 40, pp. 1201-1215, 1997.

[9] J. Laneau, "When the deaf listen to music: pitch perception with cochlear implants," Phd dissertation, Katholieke Universiteit Leuven, Faculteit Toegepaste Wetenschappen, May 2005.

[10] R. P. Carlyon and J. M. Deeks, "Limitations on rate discrimination," Journal of the Acoustical Society of America, vol. 112, pp. 1009-1025, 2002.

[11] L. Geurts and J. Wouters, "Coding of the fundamental frequency in continuous interleaved sampling processors for cochlear implants," Journal of the Acoustical Society of America, vol. 109, pp. 713-726, 2001.

[12] Q-J. Fu, F-G. Zeng, R. V. Schannon, and S. D. Soll, "Importance of tonal envelope cues in chinese speech recognition," Journal of the Acoustical Society of America, vol. 104, no. 1, pp. $505-510,1998$.

[13] Boston Scientific, HiRes with Fidelity 120 Sound Processing, A report from Advanced Bionics, the Auditory Business of Boston Scientific, September 1992

[14] Z. M. Smith, B. Delgtte, and A. J. Oxenham, "Chimeric sounds reveal dichotomies in auditory perception," Nature, vol. 416, pp. 87-90, 2002.

[15] O.Macherey, R. Carlyon, O. A. vanWieringen, and J.Wouters, "A dual-process integrator-resonator model of the electrically stimulated human auditory nerve," Journal of the Association for Research in Otolaryngology, vol. 8, no. 1, pp. 84-104, 2007

[16] Lassaletta L, Castro A, Bastarrica M, Pérez-Mora R, Madero R, De Sarriá J, Gavilán J. "Does music perception have an impact on quality of life following CI implantation?," (Acta Otolaryngol. 2007 Jul;127(7):682-6.

[17] http://cirrie.buffalo.edu/encyclopedia/en/article/293/

[18] D. Pressnitzer, J. Bestel, and B. Fraysse, "Music to electric ears: Pitch and timbre perception by CI patients," Annals new york academy of sciences, vol. 1060, pp.343-345, 2005.

[19] M. C. Leal, Y. J. Shin, M. Laborde, M. Calmels, S. Verges, S. Lugardon, S. Andrieu, O. Deguine, and B. Fraysse, "Music perception in adult cochlar implant recipients," ActaOto-Laryngologica, vol. 123, , 2003.

[20] K. Gfeller, S. Witt, J. Stordahl, M. Mehr, and G. Woodworth, "Perception of rhythmic and sequential pitch patterns by normally hearing adults and adult CI users," Ear and Hearing, vol. 18, pp. 252-260, 1997.

[21] R. P. Carlyon and J. M. Deeks, "Limitations on rate discrimination," Journal of the Acoustical Society of America, vol. 112, pp. 1009-1025, 2002.
[22] S. Pijl and D.W.F. Schwarz, "Melody recognition an musical interval perception by deaf subjects stimulated with electrical pulse trains through single CI electrodes, "Journal of the Acoustical Society of America, vol. 98, no. 2, pp. 886-895, 1998.

[23] R. Kang, G.L. Nimmons, W. Drennan, J. Longnion, C. Ruffin, K. Nie, J.H. Won, T. Worman, B. Yueh, J. Rubinstein, ,Development and validation of the University of Washington Clinical Assessment of Music Perception test" Ear Hear. 2009 Aug;30(4):411-8.

[24] B. Townshend, N. Cotter, D. van Compernolle, and R. L. White, "Pitch perception by cochlear implant subjects," Journal of the Acoustical Society of America, vol. 82, pp. 106-115, 1987.

[25] B. S.Wilson, D. T. Lawson,M. Zerbi, and C. C. Finley, "Recent developments with the CIS strategies," HochmairDesoyer of the Third International Cochlear Implant Conference, pp. 103-112, April 1993.

[26] G S. Donaldson and H. A. Kreft, "Place-pitch discrimination of single- versus dual- electrode stimuli by cochlear implant users," Journal of the Acoustical Society of America, vol. 118 , no. 2, pp. 623-626, 2005.

[27] D. B. Koch, M. Downing, M. J. Osberger, and L. Litvak, "Using current steering to increase spectral resolution in CII and HiRes 90k users," Ear and Hearing, vol. 28, no. 2, pp. 38-41, April 2007.

[28] H. J. McDermott and C. M. McKay, "Pitch ranking with non-simultaneous dual-electrode electrical stimulation of the cochlea," Journal of the Acoustical Society of America, vol. 96, no. 1, pp. 451-515, 1994.

[29] Ben H. Bonhama, Leonid M. Litvak," Current focusing and steering: Modeling, physiology, and psychophysics," Hearing Research 242 (2008) 141-153.

[30] A. G. Srinivasan, D. M. Landsberger, R. V. Shannon, "Current focusing sharpens local peaks of excitation in cochlear implant stimulation", Hearing Research, 270 89100,2010

[31] O. Macherey, J. M. Deeks, R. P. Carlyon, "Extending the limits of place and temporal pitch perception in cochlear implant users", JARO, 12 233-251, 2011.

[32] Saoji AA, Litvak LM, "Use of "phantom electrode" technique to extend the range of pitches available through a CI," Ear Hear. 2010 Oct; 31(5):693-701.

[33] A. J. Alexander, L. Bartel, L. Friesen, D. Shipp, J. Chen, "From fragments to the whole: a comparison between CI users and normal-hearing listeners in music perception and enjoyment, J Otolaryngol Head Neck Surg. 2011.

[34] W. Nogueira, L. M. Litvak, B. Edler, J. Ostermann, and A. Büchner, "Signal Processing Strategies for Cochlear Implants Using Current Steering," EURASIP Journal on Advances in Signal Processing, vol. 2009, Article ID 531213, 20 pages, 2009.

[35] W. Nogueira, A. Foerstel, A. Saoji, L. Litvak, V. Hamacher, T. Rottmann, Th. Lenarz, A. Buechner, „Evaluation of a new signal processing strategy including a partial bipolar channel to transmit low frequency information,", CIAP 2011, 20011 
